\title{
Caracterización de la Formación Académica en Emprendimiento de Pregrado que se realiza en Universidades Chilenas
}

\author{
Raúl A. Herrera y Marco A. Villalobos* \\ Universidad de Tarapacá, Escuela Univ. de Ing. Indus., Inf. y Sis., Especialidad de Ingeniería en \\ Computación e Informática, Casilla 7-D, Arica-Chile
}

* Autor a quien debe ser enviada la correspondencia

Recibido Nov. 20, 2017; Aceptado Ene. 17, 2018; Versión final Feb. 15, 2018, Publicado Ago. 2018

\begin{abstract}
Resumen
En este artículo, se presenta un estudio exploratorio para caracterizar las iniciativas formativas en emprendimiento en universidades chilenas. La muestra correspondió a universidades que respondieron a una convocatoria de participación y que entregaron información sobre sus iniciativas. En dichas instituciones, se realizaron una serie de entrevistas a distintos actores relevantes en el proceso formativo y también se realizaron visitas a las dependencias donde dicho proceso se lleva a cabo. Esto permitió recolectar información para describir el avance de las iniciativas en cada una de ellas. Se puede observar en los resultados que no hay correlación entre el grado de implementación de las respectivas iniciativas y la ubicación geográfica de la institución. Se concluye que dichas instituciones poseen distintos grados de logro en la implementación de procesos formativos en emprendimiento, que van desde un grado incipiente a un grado de madurez y solvencia para sustentar y mejorar el proceso formativo.
\end{abstract}

\section{Characterization of the Undergraduate Academic Formation in Entrepreneurship performed in Chilean Universities}

\begin{abstract}
In this paper an exploratory study to characterize the entrepreneurship's formative initiatives in Chilean universities is presented. The sample belongs to universities that responded to a participation calling and that reported information about their initiatives. On those institutions, a series of interviews to relevant actors on the formative process were performed and also visits to the institutions where that formative process is done were carried out. This allowed collecting t information to describe the advance of the initiatives in each one of them. It can be observed in the results that there is no correlation between the degree of implementation of the initiatives and the geographic location of the institutions. It is concluded that those institutions have different degrees of achievement on the implementation of entrepreneurship's formative processes, from an initial level to a degree of maturity and solvency to support and improve the formative process.
\end{abstract}

Keywords: formative-proposal; entrepreneurship; engineering; universities. 


\section{INTRODUCCIÓN}

En las Escuelas de Ingeniería de la Universidad de Tarapacá (UTA), Arica, Chile, se encuentran dificultades en el desarrollo de iniciativas formativas en emprendimiento. Esto se debe a que no se ha institucionalizado un proceso de formación emprendedora, ni se ha cimentado un adecuado acompañamiento que permita el desarrollo y continuidad de iniciativas emprendedoras dentro de los planes de estudios de las carreras de Ingeniería en la Universidad. Actualmente, los esfuerzos se han concentrado en la creación de iniciativas aisladas y desarticuladas, existiendo un Centro de Desarrollo de Negocios que es una iniciativa PúbicoPrivada no orientada a la formación en emprendimiento. Además, se evidencia la ausencia de incentivos que permitan promocionar iniciativas formativas en emprendimiento que sustenten el desarrollo de proyectos, por parte de estudiantes que de alguna manera constituyan un factor importante para la creación y consolidación de ideas de negocio en las carreras de Ingeniería.

Para enfrentar estas dificultades, se diseñó una estrategia que contempla en una primera instancia, un análisis de la realidad nacional para conocer cómo las distintas universidades están afrontando el desafío de la formación en emprendimiento de sus alumnos de pregrado (se entenderá por pregrado, como un programa académico conducente a un título profesional universitario), en particular en ingeniería, para posteriormente, proponer un enfoque formativo para alumnos de la UTA en dicha especialidad. Así, se ideó un plan de visitas a diferentes universidades nacionales, para caracterizar la formación de alumnos de pregrado en emprendimiento, conociendo in situ los diversos enfoques y buenas prácticas empleadas para estos efectos. Se entrevistó a actores relevantes, se realizaron los recorridos por las dependencias y se recolectó evidencia y resultados de las respectivas instituciones visitadas. Cabe mencionar que el plan inicialmente fue concebido para conocer la realidad en las carreras de ingeniería, pero en general, en el trabajo de campo, las instituciones describieron sus iniciativas de manera global, lo que se recogió en un indicador denominado "nivel" cuyos valores pueden ir desde institucional a profesor/curso. Por esta razón, la presentación de los resultados en este artículo es de manera general por institución.

De los análisis, se concluye que, en general, las universidades chilenas de la muestra, han realizado esfuerzos para implementar iniciativas de formación en emprendimiento; sin embargo, los resultados de estas iniciativas presentan una heterogeneidad en su implementación y logros, reflejándose en que algunas de ellas se encuentren en extremos opuestos. De esta manera, el estado de implementación de iniciativas de formación en emprendimiento de las universidades visitadas se puede clasificar en cuatro categorías: institucionalizado, es decir, la institución posee capacidad y madurez para mantener y mejorar su proceso formativo; implementado, es decir, la institución posee iniciativas formativas en marcha, pero sin garantías de su mantención en tiempo y no asegura el logro de todos los aspectos de dicho proceso; en vías de implementación, es decir, existen iniciativas emergentes o a nivel de definición; e inexistente o incipiente, es decir, las universidades en este estado poseen iniciativas aisladas o no tienen. A nivel nacional, entidades e iniciativas tales como ProChile (Dirección de Promoción de Exportaciones de Chile), Corfo (Corporación de Fomento de la Producción de Chile), Asech (Asociación de Emprendedores de Chile), Startup-Chile (Aceleradora de negocios del Gobierno de Chile) e Innovo (Centro de innovación y transferencia tecnológica, Universidad de Santiago de Chile), y a nivel mundial tales como GEM (Global Entrepreneurship Monitor) y la OCDE (Organización para la Cooperación y el Desarrollo Económicos), señalan que el emprendimiento es el motor para el desarrollo de las naciones (Bucardo et al., 2015; Galindo et al., 2016) y que éste debe ser incorporado a la formación de profesionales (Ramos y Bayter, 2012) especialmente en el área de la ingeniería (Proyecto Corfo Ingeniería 2020 y 2030).

El fomento y la difusión de la cultura emprendedora universitaria se ha convertido en un elemento clave para generar impacto social y económico, a través de los egresados (Contreras-Velásquez et al., 2017; Sánchez y Ros, 2014), pero como lo indican Priegue et al. (2014) algunos de ellos presentan dificultades para lograr sus objetivos de emprendimiento y laborales. Esto requiere que las universidades diseñen procesos formativos de emprendimiento adecuados, incluyendo sistemas basados en el uso de recursos computacionales para la enseñanza en emprendimiento (Cooper, 2007) y estrategias de desarrollo de habilidades personales, como la creatividad (Morrison y Johnston, 2003). Así, el emprendimiento universitario requiere de la correcta definición, implementación e institucionalización de un proceso formativo. En este sentido, se entiende que un proceso de este tipo comprende la respuesta a distintas preguntas fundamentales como el para qué, qué, quién y cómo se lleva a cabo. En la tabla 1, se muestran la diferencia entre los enfoques tradicionales y emergentes para responder a estas cuestiones del emprendimiento universitario, presentes en el trabajo de Siegel y Wright (2015).

También, el proceso formativo, incluye los aspectos educativos tales como un conjunto de actividades curriculares teórico-práctico (García-Rodríguez et al. 2016; Ruskovaara y Pihkala, 2013; Riese, 2011), método de enseñanza-aprendizaje, evaluación y los instrumentos necesarios, en base a las necesidades de los distintos stakeholders (Omer Attali y Yemini, 2017). En este sentido, este trabajo pretende caracterizar la 
formación de alumnos de pregrado en emprendimiento de universidades chilenas, conociendo in situ los diversos enfoques y buenas prácticas empleadas para estos efectos. A partir de dicha caracterización, se propondrá un enfoque formativo para alumnos de las carreras de ingeniería de la UTA.

Tabla 1: Enfoques tradicional y emergente del emprendimiento universitario (Siegel y Wright, 2015)

\begin{tabular}{|l|l|l|}
\hline Aspecto & \multicolumn{1}{|c|}{ Enfoque tradicional } & \multicolumn{1}{c|}{ Enfoque Emergente } \\
\hline Para qué & $\begin{array}{l}\text { Generar retorno financiero } \\
\text { directo }\end{array}$ & $\begin{array}{l}\text { Proveer un beneficio social y económico más amplio al ecosistema de la } \\
\text { universidad }\end{array}$ \\
\hline Qué & $\begin{array}{l}\text { Spin-offs académicos; } \\
\text { licenciamientos, patentes. }\end{array}$ & $\begin{array}{l}\text { Start-ups de estudiantes y ex alumnos; Estudiantes formados en } \\
\text { emprendimiento; creación de trabajo en la región o localidad. }\end{array}$ \\
\hline Quién & $\begin{array}{l}\text { Facultades académicas y } \\
\text { post doctorados }\end{array}$ & $\begin{array}{l}\text { Estudiantes; ex alumnos, industrias colaboradoras dentro de los campus; } \\
\text { emprendedores tutores o mentores. }\end{array}$ \\
\hline Cómo & $\begin{array}{l}\text { Oficinas de transferencia } \\
\text { tecnológica; parques } \\
\text { científicos }\end{array}$ & $\begin{array}{l}\text { Aceleradoras; taller (físico) de emprendimiento; competiciones de planes } \\
\text { de negocios estudiantiles; redes de colaboración con la industria y ex } \\
\text { alumnos; } \\
\text { Movilidad laboral; incubadoras público-privadas. }\end{array}$ \\
\hline
\end{tabular}

\section{REVISIÓN DE LA LITERATURA}

Un estudio prospectivo sobre primeras iniciativas de educación en emprendimiento en las universidades de Estados Unidos (1876 a 1999), realizado por Katz (2003), mostró que este tipo de formación debería ser incorporada en el currículo ya que ella generaría riqueza y crecimiento para el país (pp. 16). Es por ello que indicó además, la necesidad de desarrollar una infraestructura adecuada y una evaluación del impacto real de las iniciativas. Así mismo, identificó problemas y desafíos para las décadas siguientes al estudio. Sin embargo, como lo señala el autor, estudios de esta naturaleza (sólo bibliográfico), puede generar ciertos sesgos y la carencia de comprobación empírica de ciertos resultados y prácticas.

El trabajo de Guerrero et al. (2014), realiza una comparación entre universidades que forman alumnos emprendedores de dos regiones diferentes de Europa, España (2) e Irlanda (2), haciendo dicha comparación a nivel de la economía institucional y los recursos. Este estudio se llevó a cabo entre el 2006 y 2010 , donde se adoptó la metodología de múltiples casos de estudio, para explorar las diferencias principales dentro de los factores condicionantes de resultados y salidas de estas regiones, caracterizando las universidades en base a la región donde se encuentran, tipo, año de fundación, ranking y características distintivas de emprendimiento. El análisis se centró principalmente en informes de resultados y publicaciones realizadas por dichas universidades y recolección de otros parámetros de funcionamiento interno. Este estudio permitió establecer factores comunes entre ambas regiones, sobre todo en lo relativo a interacción con el medio productivo y la importancia de una estructura organizacional y financiamiento que apoye directamente el contacto y fortalecimiento con el ambiente emprendedor.

El trabajo de Lee et al. (2005), presenta una comparación del impacto de la educación en emprendimiento en Estados Unidos y Korea, donde se analiza como el contexto cultural influye en los resultados de dichas iniciativas. En este estudio, se analizaron los resultados de la experiencia educativa en emprendimiento para 2 universidades (University of Nebraska-Lincoln y Kyonggi University, respectivamente). Los resultados mostraron que para estudiantes de ambos países, la educación en emprendimiento y una infraestructura que lo sustente, son de vital importancia en sus futuros profesionales, independiente de las diferencias culturales entre ambos países.

En el trabajo de Sánchez et al. (2017) se describen distintas iniciativas académicas en nivel mundial, indicando las buenas prácticas que se han aplicado. Su investigación, consideró una recopilación de artículos científicos que le permitieron evidenciar la realidad actual sobre la educación emprendedora, es decir, ella debería considerar los desafíos tales como la competencia internacional y las tecnologías (pp. 28). Para lograr este objetivo, los autores indican que se recopiló información proveniente de 107 fuentes investigativas, refiriéndose a libros y artículos de revista. Para evaluar el estado actual de la educación en los países latinoamericanos, se utilizaron fuentes que tuvieran menos de 20 años, de las cuales los programas investigados fueron verificados para corroborar su función y vigencia. Las bases de datos utilizadas para consultar la literatura fueron ProQuest, Scopus, además de reportes o libros realizados por agencias gubernamentales de ministerios de educación, la Comisión Europea y el GEM. En Arnaut (2010) y Nwaogu (2014), se describen las características de las universidades que se denominan emprendedoras, es decir, universidades que han definido en su misión la educación en emprendimiento como un eje importante en su quehacer. 
A nivel nacional, un interesante estudio descrito por Romaní et al. (2016), fue realizado por el Centro de Emprendimiento y de la Pyme de la Universidad Católica del Norte Sede Antofagasta que se refiere a la intención emprendedora y actividad de sus estudiantes. El estudio entrega información sobre el rol de la educación en la formación emprendedora a nivel superior de la UCN, mostrando datos sobre la actitud, actividad, intención y aspiración emprendedora de los estudiantes de dicha casa de estudios. Finalmente, en los trabajos de Soria et al. (2016); Contreras-Velásquez et al. (2017); y Delicio (2006), se presentan sendos casos particulares de iniciativas de formación universitaria en emprendimiento, donde se evidencia la importancia de estos programas educativos y su impacto en el desarrollo económico de un país. Es importante destacar que en general los estudios reportados tienen como fuentes de datos reportes, artículos científicos, libro, etc. Y por otro lado, a nivel nacional, no existen estudios generales in situ de cómo las universidades están desarrollo iniciativas formativas en emprendimiento, por ejemplo: recursos, políticas y normativas, estructuras organizativas, reconocimientos e incentivos, instancias para socializar emprendimientos y soporte curricular. En este sentido, este trabajo presenta la caracterización de la formación de alumnos de pregrado en emprendimiento de universidades chilenas, conociendo in situ los diversos enfoques y buenas prácticas empleadas para estos efectos.

\section{MATERIALES Y MÉTODOS}

El trabajo de investigación tuvo como filosofía el positivismo (el conocimiento es concreto y los fenómenos son factibles de medición), como enfoque, el deductivo a través de confrontación teórica y empírica, el estudio fue de naturaleza transversal y el método de recolección de datos primarios fue a través de la aplicación de una entrevista semiestructurada a actores relevantes de varias universidades chilenas, en el sector de la formación en emprendimiento, durante el mes de octubre de 2017. Fundamentalmente fue una investigación exploratoria.

\section{Objetivo}

El objetivo de este trabajo fue caracterizar la formación de alumnos de pregrado en emprendimiento de universidades chilenas, principalmente en carreras de ingeniería, conociendo in situ los diversos enfoques y buenas prácticas empleadas para estos efectos.

\section{Problema y pregunta de investigación}

En las Escuelas de Ingeniería de la UTA, se encuentran dificultades en el desarrollo de iniciativas formativas en emprendimiento, por ejemplo: recursos, políticas y normativas, estructuras organizativas, reconocimientos e incentivos, instancias para socializar emprendimientos y soporte curricular. Esto se debe a que no se ha institucionalizado un proceso de formación emprendedora, ni se ha cimentado un adecuado acompañamiento que permita el desarrollo y continuidad de iniciativas dentro de los planes de estudios de las carreras de Ingeniería en la Universidad. Actualmente, los esfuerzos se han concentrado en la creación de iniciativas aisladas y desarticuladas, existiendo un Centro de Desarrollo de Negocios que es una iniciativa PúbicoPrivada no orientada a la formación en emprendimiento, sino que al apoyo de iniciativas ya estructuradas, sean internas o externas a la Institución. Además, se evidencia la ausencia de incentivos que permitan promocionar iniciativas formativas en emprendimiento que sustenten el desarrollo, por parte de estudiantes, proyectos de corto, mediano y largo plazo que de alguna manera constituyan un factor importante para la creación y consolidación de ideas de negocio en las carreras de ingeniería.

Teniendo en cuenta lo anterior, se desprendió una cuestión principal ¿Cuál es el proceso que podría permitir la formación en emprendimiento que considere recursos, políticas y normativas, estructuras organizativas, reconocimientos e incentivos, instancias para socializar emprendimientos, soporte curricular y herramientas académicas para los estudiantes de las Escuelas de Ingeniería de la UTA?. Para responder esta interrogante, se diseñó un plan de acción que contempla en una primera instancia, un análisis de la realidad nacional para conocer cómo las distintas universidades están afrontando el desafío de la formación en emprendimiento de sus alumnos de pregrado, en particular en ingeniería, para posteriormente, proponer un enfoque formativo para alumnos de nuestra institución en dicha especialidad. Este trabajo presenta los resultados obtenidos de la ejecución de la primera parte del plan de acción.

\section{Diseño conceptual}

La investigación, fue planificada y realizada durante los meses de marzo a octubre del 2017, por la necesidad de caracterizar la formación de alumnos de pregrado en emprendimiento de universidades chilenas, para posteriormente hacer una propuesta formativa propia. Con este objetivo, se diseñó el modelo de investigación que se muestra en la fig. 1. 
Como primer paso, en una definición del contexto de la investigación, se establecieron los objetivos del proceso de caracterización, el problema y la pregunta de investigación, se seleccionó el tipo de instrumento, se definieron las secciones que contendría el guion de la entrevista semiestructurada, se realizó una selección de las universidades a ser visitadas y finalmente, se construyó un plan de visitas. En el segundo paso, en un trabajo de campo, se desarrollaron las entrevistas planificadas (declaración de actores), se realizaron los recorridos por las instalaciones físicas (observación in situ) y la recolección de evidencias y resultados de las respectivas instituciones visitadas (análisis de texto). En un tercer paso, se llevó a cabo un análisis de resultados del trabajo (análisis de contenido), partiendo por una definición de criterios comparativos y la construcción de una matriz de los datos resultantes del análisis de acuerdo a los criterios y que se encontraban registrados en los medios audiovisuales. Finalmente, se analizó la evidencia recolectada (indicadores de resultado), incorporándose al análisis general.

\section{Instrumentos}

Se discutió sobre los diversos métodos para la recolección de la información requerida. Considerando que el estudio es de tipo exploratorio donde se pueda recabar tanto información cualitativa como cuantitativa, se decidió entrevistar a actores relevantes en el desarrollo de iniciativas formativas en emprendimiento. Posteriormente, se analizó entre las 3 formas de entrevistas posibles, eligiéndose la entrevista semiestructurada debido a su flexibilidad y a la capacidad que ofrece para orientar al entrevistado hacia el logro del objetivo y explorar líneas de información no consideradas inicialmente en el guión.

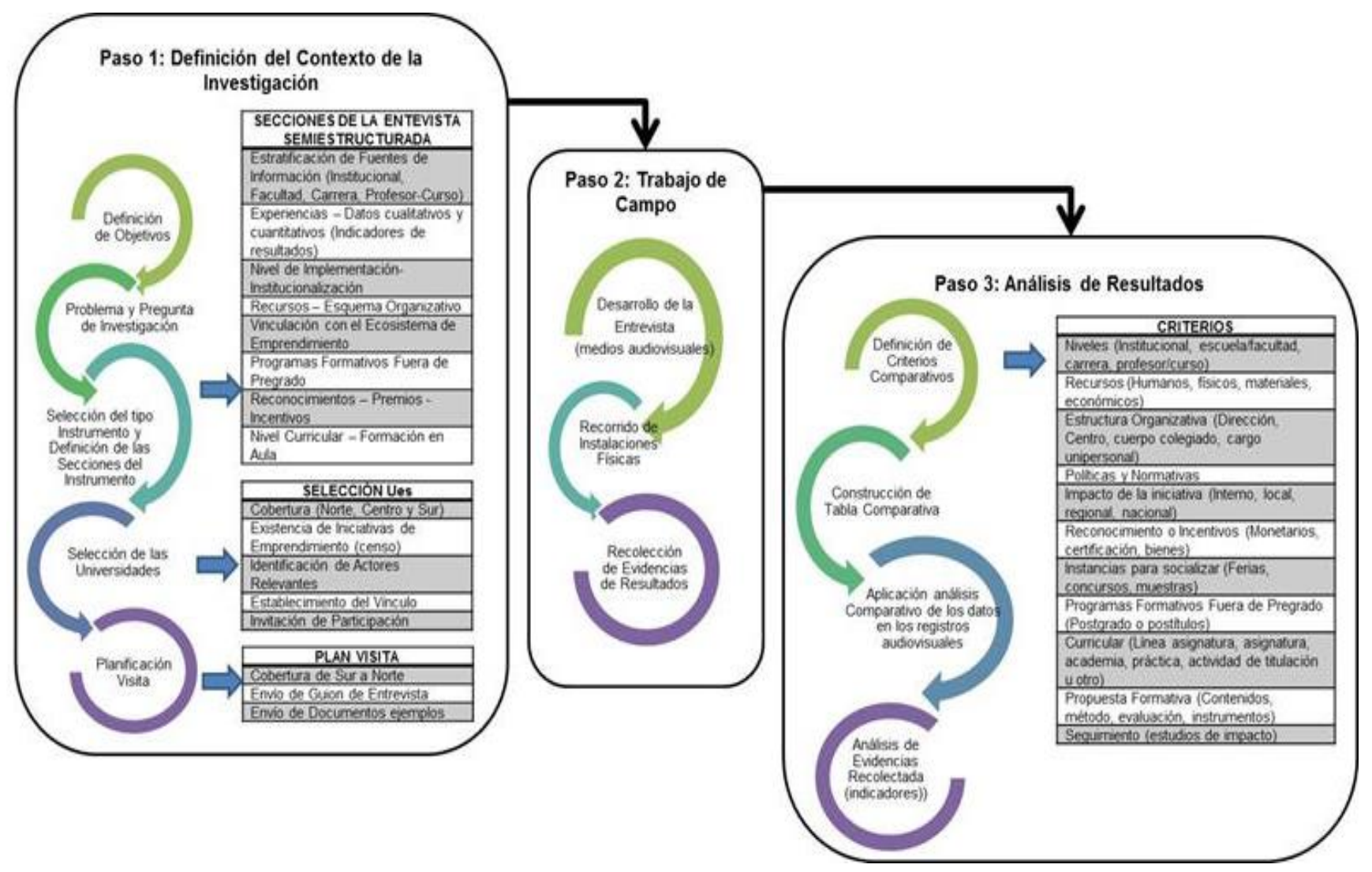

Fig. 1: Modelo Conceptual de la Investigación

La construcción del guion de la entrevista semiestructurada consideró en primer lugar cubrir las distintas preguntas fundamentales como el para qué, qué, quién y cómo se llevan a cabo los procesos formativos en las distintas universidades. También, incluir qué actividades curriculares teórico-práctico, método de enseñanza-aprendizaje, evaluación y cuáles son los instrumentos que son utilizados. Así, finalmente, el guion consideró los criterios de estratificación, experiencias realizadas, nivel de implementación e institucionalización, recursos, esquema organizativo, vinculación con el ecosistema de emprendimiento, programas formativos fuera de pregrado, reconocimientos y nivel curricular, como se muestran en la fig. 1 sección paso 1.

El instrumento se validó mediante su envío previo a los actores relevantes que contestaron la invitación para ser entrevistados, solicitándoles que tomaran conocimiento e hicieran las observaciones que consideraran pertinentes. Por otra parte, para el análisis de contenido de las entrevistas semiestructuras y los documentos recopilados, se construyó una matriz de análisis documental comparativa. 


\section{Técnica de análisis de datos}

Como se puede observar en la fig. 1 sección paso 3, la técnica de análisis de datos consideró en primer lugar, la confección de una matriz comparativa que contiene los mismos aspectos que usaron para la construcción de la entrevista. En dicha matriz, se transfirieron los datos registrados en el material audiovisual obtenido en cada entrevista, aplicando un análisis sintáctico, semántico y pragmático (Ballester, 2006). Posteriormente, se agregaron al análisis los datos provenientes de la evidencia recolectada (informes, material promocional, normativas, etc.), mediante el análisis de texto. Hasta aquí, la matriz contiene datos tanto cualitativos como cuantitativos, así que se normalizó, valorando los aspectos-criterios-sub criterios como se indica en la tabla 2. Los criterios evaluados de 1 a 4 son acumulativos, es decir, una institución, puede alcanzar todos los subcriterios (sumando 10). Por su parte los evaluados con 0, 5 o 10, son exclusivos, es decir, una institución, puede poseer solo uno de esos valores para el subcriterio.

\section{Determinación y descripción de la muestra}

Se utilizó una muestra intencionada por disponibilidad. En primer lugar, se seccionó el territorio nacional en tres grandes subsectores (norte, centro y sur). Posteriormente, se exploró la existencia de iniciativas de formación en emprendimiento dentro de las universidades en cada uno de los subsectores. En cada una de ellas se identificaron actores relevantes y se les envió una solicitud de colaboración para la investigación vía correo electrónico. Cabe destacar que todas las universidades visitadas imparten carreras de Ingeniería. En la tabla 3, se puede apreciar una descripción de las universidades visitadas. Esta tabla fue elaborada por los autores, pero las características corresponden al estudio realizado por Rivas et al. 2014. Se destaca el promedio de años de acreditación ( 5.5 años) y el promedio de la posición del ranking de nacional emitido por el Ministerio de Educación de Chile (posición promedio 16).

Tabla 2: Valoración de aspectos-criterios-sub criterios para el análisis comparativo

\begin{tabular}{|c|c|c|c|}
\hline Aspectos & Criterios & Sub criterios & Valoración \\
\hline \multirow{8}{*}{ Para qué } & \multirow{4}{*}{ Niveles } & Institucional & 4 \\
\hline & & Escuela/facultad & 3 \\
\hline & & Carrera & 2 \\
\hline & & Profesor/curso & 1 \\
\hline & \multirow{4}{*}{ Impacto de la iniciativa } & Interno & 4 \\
\hline & & Local & 3 \\
\hline & & Regional & 2 \\
\hline & & Nacional & 1 \\
\hline \multirow{9}{*}{ Qué } & \multirow{3}{*}{ Programas fuera pregrado } & Sí & 10 \\
\hline & & Sí, pero escasos & 5 \\
\hline & & No & 0 \\
\hline & \multirow{4}{*}{ Curricular } & Línea asignatura & 4 \\
\hline & & Asignatura & 3 \\
\hline & & Academia & 2 \\
\hline & & Otro & 1 \\
\hline & \multirow{2}{*}{ Propuesta Formativa } & Sí & 10 \\
\hline & & No & 0 \\
\hline \multirow{3}{*}{ Quién } & \multirow{3}{*}{ Seguimiento } & Sí & 10 \\
\hline & & Sí, pero escaso & 5 \\
\hline & & No & 0 \\
\hline \multirow{16}{*}{ Cómo } & \multirow{3}{*}{ Recursos } & Sí & 10 \\
\hline & & Sí, pero escasos & 5 \\
\hline & & No & 0 \\
\hline & \multirow{4}{*}{ Estructura Organizativa } & Dirección & 4 \\
\hline & & Centro & 3 \\
\hline & & Cuerpo colegiado & 2 \\
\hline & & Cargo unipersonal & 1 \\
\hline & \multirow{3}{*}{ Políticas y Normativas } & Sí & 10 \\
\hline & & Sí, pero escasa & 5 \\
\hline & & No & 0 \\
\hline & \multirow{3}{*}{ Reconocimiento o Incentivo } & Sí & 10 \\
\hline & & Sí, pero escaso & 5 \\
\hline & & No & 0 \\
\hline & \multirow{3}{*}{ Instancias para socializar } & Sí & 10 \\
\hline & & Sí, pero escasas & 5 \\
\hline & & No & 0 \\
\hline
\end{tabular}


Tabla 3: Descripción de la muestra de universidades (elaborada con base en Rivas et al. 2014)

\begin{tabular}{|l|l|c|}
\hline \multicolumn{1}{|c|}{ Característica } & \multicolumn{1}{|c|}{ Descripción } & Cantidad \\
\hline \multirow{2}{*}{ Ubicación Geográfica } & Norte & 4 \\
\cline { 2 - 3 } & Centro & 5 \\
\cline { 2 - 3 } & Sur & 2 \\
\hline Calidad Institucional & Promedio de años de acreditación (2 a 7 años) & 5.5 \\
\hline \multirow{2}{*}{$\begin{array}{l}\text { Propiedad y } \\
\text { aporte fiscal }\end{array}$} & Estatal & 2 \\
\cline { 2 - 3 } & Privada con subsidio & 8 \\
\cline { 2 - 3 } & Sin aporte estatal & 1 \\
\hline Ranking (Mineduc, Chile, 2016) & Promedio posición (1 - 45) & $19,6 \%$ \\
\hline $\begin{array}{l}\text { Porcentaje respecto al universo de } \\
\text { universidades nacionales (Mineduc, } \\
\text { Chile, 2017) }\end{array}$ & Universo 56 - Visitadas 11 & \\
\hline
\end{tabular}

\section{RESULTADOS Y DISCUSIÓN}

Los resultados de la experiencia han sido muy positivos en todos los aspectos, lográndose los objetivos planteados. Se conocieron las distintas propuestas formativas en emprendimiento de 11 instituciones universitarias, correspondiendo a un $20 \%$ aproximadamente de las universidades nacionales. Es importante destacar que luego de los análisis, no hay relación entre la zona geográfica donde se ubican las instituciones (norte, centro o sur del país) y la calidad y cantidad de aspectos asociados con una iniciativa formativa en emprendimiento. De esta manera, la matriz resultante no reflejó ningún orden por zona geográfica. Un primer conjunto de resultados se pueden desprender desde el la fig. 2 donde se describen los puntajes obtenidos por cada institución, luego de la aplicación del método de análisis.

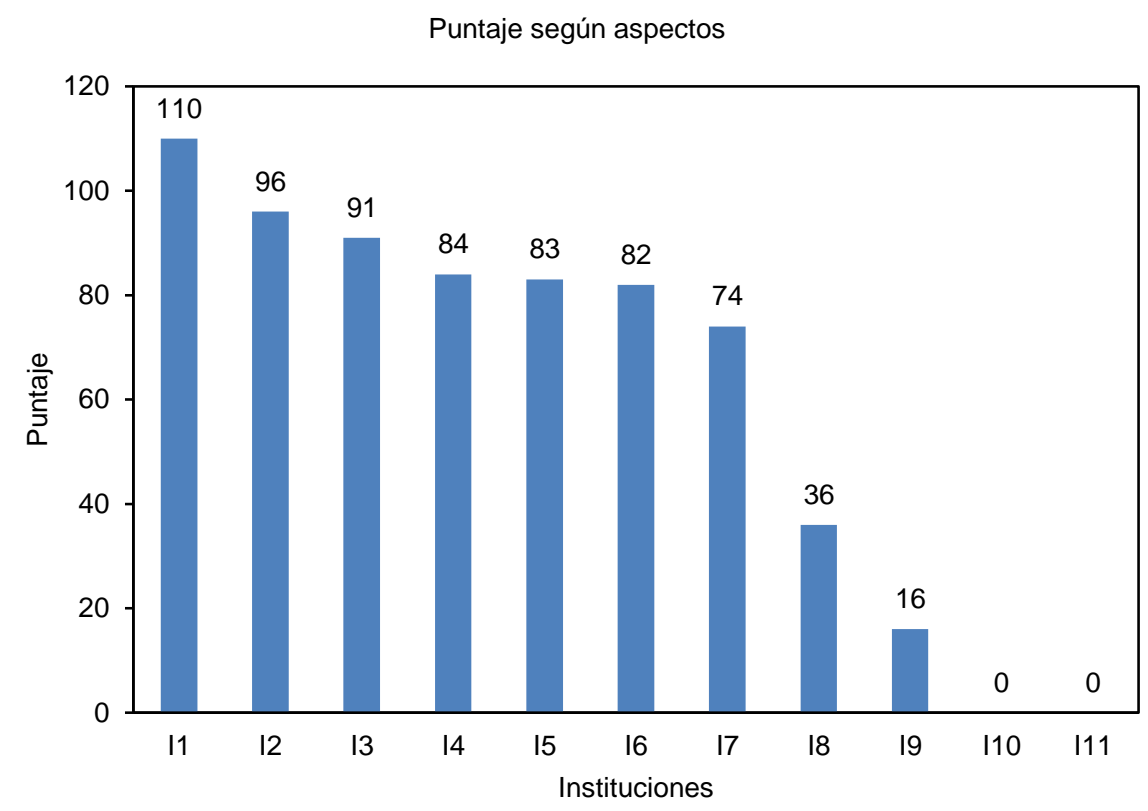

Fig. 2: Puntajes obtenidos por cada institución

Así, en la fig. 2, se puede observar que el promedio de las universidades (61,1 puntos de 110) indica que en términos generales, ellas poseen iniciativas en emprendimiento que implementan la mayoría de los aspectos. También, se puede observar que existe una heterogeneidad dentro de la muestra de universidades, ya que sobre el promedio existen instituciones que han logrado la mayoría de los criterios y con altos valores, pero también existen instituciones que no han logrado el cumplimiento de los criterios o hasta la inexistencia de iniciativas de emprendimiento. Finalmente, como ya se mencionó, se puede indicar que los resultados son independientes de la zona geográfica de la institución.

Un segundo conjunto de resultados se pueden desprender desde el la fig. 3 donde se describen los puntajes promedios obtenidos por la muestra de instituciones en los criterios, recordando que ellos son evaluados de 0 a 10. Por tanto, en la fig. 3, en general, se puede observar que las instituciones presentan iniciativas de emprendimiento que implementan la mayoría de los criterios. 


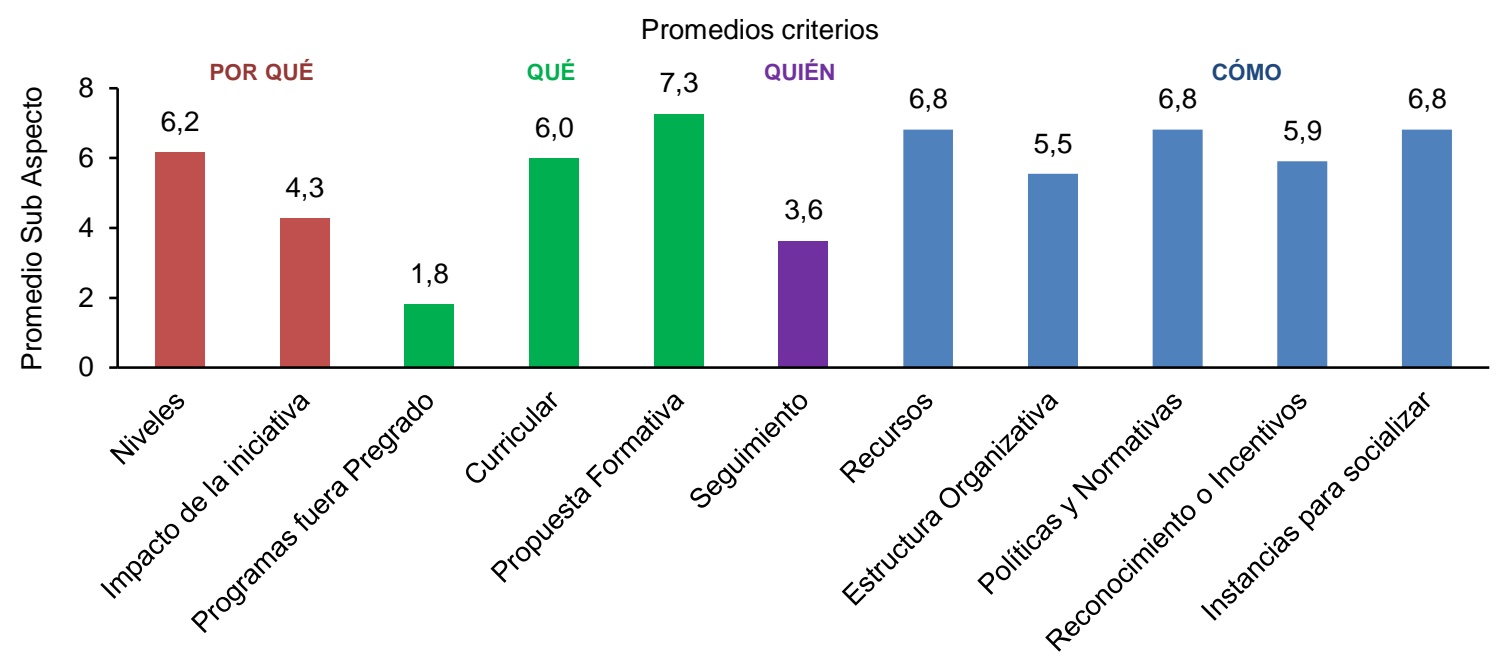

Criterios

Fig. 3: Promedios obtenidos por la muestra instituciones en los criterios

Así mismo, las instituciones presentan valores altos de logros de los sub aspectos: propuesta formativa, recursos, políticas y normativas e instancias para socializar. También, presentan valores bajos de logro en los sub aspectos: programas fuera de pregrado, seguimiento e impacto de la iniciativa. Finalmente, las instituciones visitadas, presentan un mayor logro en los sub aspectos del "Cómo", mientras que los sub aspectos menos logrados corresponden a los del "Quién". Así mismo, tanto los sub aspectos del "Por qué" como los del "Qué", presentan un logro desigual.

Finalmente, un tercer conjunto de resultados se pueden desprender desde la fig. 4, donde se describen los puntajes promedios obtenidos por la muestra instituciones en los aspectos a partir del logro de los sub aspectos respectivos. Por tanto, en la fig. 4, en general, se puede observar que las instituciones visitadas, presentan un mayor logro del aspecto "Cómo", mientras que el aspecto menos logrado es el de "Quién", consistentemente como se observó en los respectivos sub aspectos. Así mismo, tanto el aspecto "Por qué" como el "Qué", presentan un logro igual o superior a la media. También, es importante señalar que si bien, las universidades de la muestra poseen iniciativas en emprendimiento que implementan la mayoría de los aspectos, ellos se encuentran por debajo de la media (5,6 de 10 puntos), es decir, se corrobora la heterogeneidad dentro de la muestra de instituciones.

Valoración Promedio Aspecto Proceso Formativo Evaluado

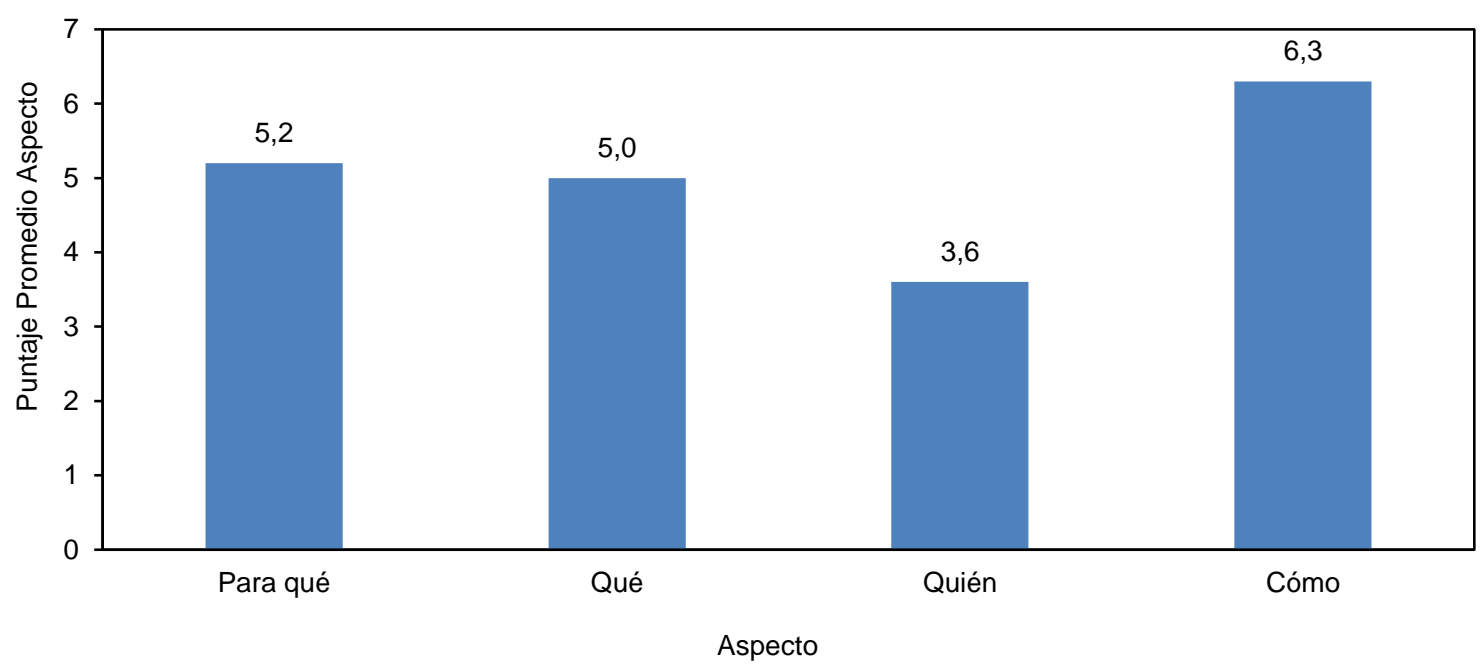

Fig. 4: Promedios obtenidos por la muestra instituciones en los aspectos

\section{DISCUSIÓN FINAL}

En general, las universidades chilenas de la muestra, han realizado esfuerzos para implementar iniciativas de formación en emprendimiento considerando recursos, políticas y normativas, estructuras organizativas, 
reconocimientos e incentivos, instancias para socializar emprendimientos y soporte curricular. Sin embargo, los resultados de estas iniciativas presentan una heterogeneidad en su implementación y logros, reflejándose en que algunas de ellas se encuentren en extremos opuestos (110 y 0). También se puede observar que las instituciones visitadas, en general, han mostrado una mayor preocupación con respecto a la realización de iniciativas formativas en emprendimiento que a su definición estratégica. Esto se evidencia por la falta de estudios de evaluación de resultados de las iniciativas, su mantenimiento en el tiempo y la ausencia de programas más allá del pregrado.

Por todo lo anterior, se puede resumir el estado de implementación de las iniciativas de formación en emprendimiento de las universidades visitadas en cuatro categorías: institucionalizado, es decir, la institución posee capacidad y madurez para mantener y mejorar su proceso formativo (puntaje promedio 99,0 de 110 correspondiendo al $27 \%$ de las universidades, y con valor promedio en el logro de los aspectos igual a 9,0 de 10); implementado, es decir, la institución posee iniciativas formativas en marcha, pero sin garantías de su mantención en el tiempo y no asegura el logro de todos los aspectos de dicho proceso (puntaje promedio 83,0 de 110 correspondiendo al $27 \%$ de las universidades, y con valor promedio en el logro de los aspectos igual a 7,5 de 10); en vías de implementación, es decir, existen iniciativas emergentes o a nivel de definición (puntaje promedio 55,0 de 110 correspondiendo al 19\% de las universidades, y con un valor promedio en el logro de los aspectos igual a 5,0 de 10); e inexistente o incipiente, es decir, las universidades en este estado poseen iniciativas aisladas o no tienen (puntaje promedio 5,3 de 110 correspondiendo al $27 \%$ de las universidades, y con valor promedio en el logro de los aspectos igual a 0,5 de 10). Las categorías indicadas en el párrafo anterior, según los resultados obtenidos, no tienen relación con la zona geográfica y el nivel de logro alcanzado por cada una las instituciones visitadas.

Por otra parte, se observó que el origen de las iniciativas formativas en emprendimiento poseen distintos inicios que van desde una motivación interna (sello institucional o iniciativas individuales); Proyectos MECESUP para definir las competencias de los perfiles de egreso, hallándose entre ellas el emprendimiento; hasta el actual proyecto CORFO Ingeniería 2030. Cabe señalar que los resultados obtenidos son coherentes con lo observado in situ. Esto es relevante, porque valida empíricamente el modelo evaluativo planteado.

Finalmente, en la actualidad se encuentra en desarrollo un modelo formativo emprendimiento con base a las buenas prácticas, resultados observados y propuestas formativas de las distintas universidades visitadas.

\section{CONCLUSIONES}

De acuerdo al trabajo presentado y a los resultados obtenidos, se pueden plantear las siguientes conclusiones principales:

1. Las universidades chilenas visitadas, poseen iniciativas de formación en emprendimiento, pero presentan una heterogeneidad en su implementación y resultados.

2. Se observó que la mayoría de las instituciones tienen una carencia de estudios de evaluación de resultados de las iniciativas de formación en emprendimiento en el tiempo y ausencia de programas más allá del pregrado que den continuidad o profundidad en el tema del emprendimiento.

3. Se observó que los resultados asociados con las iniciativas de emprendimiento, no tienen relación con la zona geográfica en la cual está inserta la institución.

4. Se observó que el origen de las iniciativas formativas en emprendimiento proviene de una motivación interna o externa.

5. Finalmente, los resultados obtenidos son coherentes con lo observado in situ, validándose el método de evaluación de las distintas iniciativas de emprendimiento realizados por las universidades chilenas visitadas.

\section{AGRADECIMIENTOS}

Este trabajo cuenta con el financiamiento de fondos para la investigación de la Universidad de Tarapacá, proyectos mayores y en educación, con códigos 8742-17 y 8722-16 respectivamente. Un reconocimiento especial a los representantes de cada institución universitaria que fueron entrevistados por su valiosa colaboración, interés y disposición. A todos y cada uno de ellos muchas gracias porque sin su colaboración esta investigación no podría haberse llevado a cabo.

\section{REFERENCIAS}

Arnaut, D., Towards an entrepreneurial University, Inter. Journal of Euro-Mediterranean Studies, 3(1), 135-152 (2010)

Ballester, L., Análisis semántico y pragmático de las entrevistas de investigación, EMPIRIA, Revista de Metodología de las Ciencias Sociales, (11), 107-129 (2006) 
Bucardo, M. A., M. Saavedra y M. Camarena, Hacia una comprensión de los conceptos de emprendedores y empresarios, Suma de Negocios, 6(13), 98-107 (2015)

Contreras-Velásquez, J., S. Wilches-Duran, M. Graterol-Rivas y M. Bautista-Sandoval, Educación Superior y la Formación en Emprendimiento Interdisciplinario: Un Caso de Estudio, Formación Universitaria, 10(3), 11-20 (2017)

Cooper, B., Central issues in the use of computer-based materials for high volume entrepreneurship education, Active Learning in Higher Education, 8(3), 201-217 (2007)

Delicio, F. A., Estrategias para el fomento del emprenderurismo universitario en la Facultad de Ciencias Económicas y Sociales de la Universidad Nacional de Mar del Plata, FACES, 12(26), 41-65, (2006)

Galindo-Martín, M.Á., M.T. Méndez-Picazo y M.S. Castaño-Martínez, Crecimiento, progreso económico y emprendimiento, Journal of Innovation \& Knowledge, 1(1), 62-68 (2016)

García-Rodríguez, F.J., C.I. Ruiz-Rosa, E. Gil-Soto y D. Gutiérrez-Taño, Promoting entrepreneurship education among university students: design and evaluation of an intervention programme, Cultura y Educación, 28(3), 565-600 (2016)

Guerrero, M., D. Urbano, J. Cunningham y D. Organ, Entrepreneurial universities in two European regions: A case study comparison, The journal of technology Transfer, 39(3), 415-434 (2014)

Katz, J.A., The chronology and intellectual trajectory of American entrepreneurship education: 1876-1999, Journal of business venturing, 18(2), 283-300 (2003)

Lee, S.M., D. Chang y S.B. Lim, Impact of entrepreneurship education: A comparative study of the US and Korea, International Entrepreneurship and Management Journal, 1(1), 27-43 (2005)

Morrison, A. y B. Johnston, Personal creativity for entrepreneurship: Teaching and learning strategies, Active learning in higher education, 4(2), 145-158 (2003)

Nwaogu, E., A Guiding Framework for Entrepreneurial Universities, EC-OECD, EU (2014)

Omer Attali, M. y M. Yemini, Initiating consensus: stakeholders define entrepreneurship in education, Educational review, 69(2), 140-157 (2017)

Priegue, D., J. García y M. Lorenzo, Higher Education and Enterprising Spirit: The Case of the Universidad de Santiago de Compostela, Procedia Social and Behavioral Sciences, 139, 48-55 (2014)

Ramos, F.S. y L.O. Bayter, Emprendimiento y economía social, oportunidades y efectos en una sociedad en transformación, CIRIEC - España, (75), 129-151 (2012)

Riese, H., Enacting entrepreneurship education: The interaction of personal and professional interests in mini-enterprises, Cambridge Journal of Education, 41(4), 445-460 (2011)

Romaní, G., M. Atienza y R. Rojas, Intención y Actividad Emprendedora de los Estudiantes de la Universidad Católica del Norte Antofagasta, Ediciones Universitarias Universidad Católica del Norte, Antofagasta, Chile (2016)

Ruskovaara, E. y T. Pihkala, Teachers implementing entrepreneurship education: classroom practices, Education + Training, 55 (2), 204-216 (2013)

Sánchez, F. y C. Ros, Development of entrepreneurial competence through practicum in pedagogy degree, ProcediaSocial and Behavioral Sciences, 139, 116-122 (2014)

Rivas, L.A., H. Gaete y X. Llinas-Audet, Segmentación y clasificación de las universidades en Chile: desventajas de inicio y efectos de las políticas públicas de financiamiento, Ingeniare, Revista chilena de Ingeniería, 22(3), 384-397 (2014)

Sánchez, J.C., A. Ward, B. Hernández y J. Florez, Educación emprendedora: Estado del Arte, DOI: http://dx.doi.org/10.20511/pyr2017.v5n2.190, Propósitos y Representaciones, 5(2), 401-473 (2017)

Siegel, D. S. y M. Wright, Academic entrepreneurship: time for a rethink? British J. of Management, 26(4), $582-595$ (2015)

Soria-Barreto, K., S. Zuniga-Jara y S. Ruiz-Campo, Educación e Intención Emprendedora en Estudiantes Universitarios: Un Caso de Estudio, doi: 10.4067/S0718-50062016000100004, Formación Universitaria, 9(1), 25-34 (2016) 